\title{
Signs of PeVatrons in Gamma-Ray Haze
}

\author{
A diffuse glow of high-energy gamma rays hints at the presence of \\ powerful cosmic accelerators, called PeVatrons, within the disk of our \\ Galaxy.
}

By Petra Huentemeyer

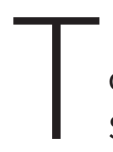

he interstellar medium is filled with relativistic charged particles-mostly protons and helium nuclei. Spaceborne telescopes and earthbound observatories have revealed that these cosmic rays can have energies exceeding $10^{15} \mathrm{eV}$ (PeV). But what are the accelerators, or "PeVatrons," that can bring particles to such high energies, and are they located in our Galaxy? Finding them could shed light on how stars evolve and on how energy is transported through the Galaxy. But despite decades of hunting, only a few PeVatron candidates have been located in the Milky Way. The Tibet AS $\gamma$ experiment, a China-Japan collaboration, has reported new measurements that provide further evidence for galactic PeVatrons. The experiment has detected a diffuse emission of gamma rays with ultrahigh energies of $0.1-1 \mathrm{PeV}$, which is likely generated by PeV cosmic rays. The spatial distribution of these

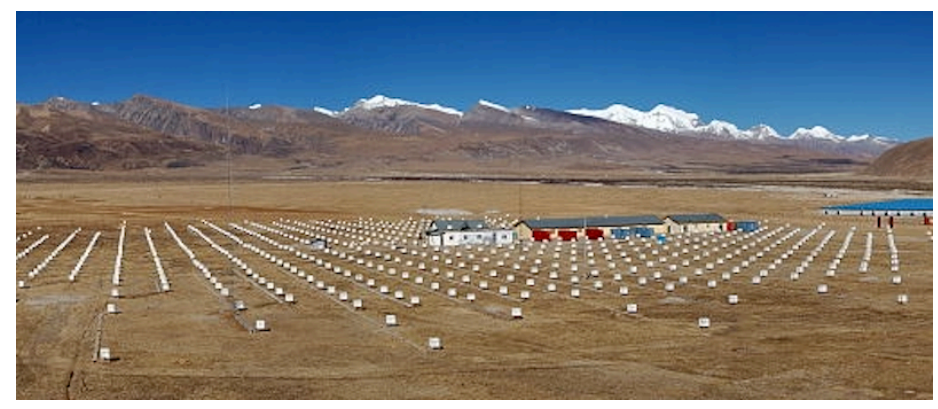

Figure 1: The $65,700 \mathrm{~m}^{2}$ array of the Tibet AS $\gamma$ experiment detects high-energy gamma rays by observing showers of particles produced when the gamma rays hit molecules in Earth's atmosphere.

Credit: Tibet AS $\gamma$ Collaboration gamma rays points at the presence of PeVatrons within our Galaxy [1].

Understanding the origin of cosmic rays is complicated by the fact that the charged particles are deflected by interstellar and intergalactic magnetic fields (with the notable exception of so-called "cosmic bullets," cosmic rays with energies approaching $10^{20} \mathrm{eV}$ ). The detection of high-energy gamma rays, however, offers a way to circumvent this problem. Such gamma rays are produced when cosmic rays collide with material, such as molecular clouds, near their production sites or on their journeys through space. These gamma rays are approximately one tenth as energetic as the parent cosmic rays. Unlike charged particles, however, gamma-ray photons aren't affected by magnetic fields. They are thus ideal carriers of information about the processes that produce high-energy cosmic rays [2].

Thanks to these features, gamma rays have allowed researchers to pinpoint a few PeVatron candidates and to test several PeVatron scenarios. Until today, the paradigmatic hypothesis has been that the principal accelerators of galactic cosmic rays are supernova remnants (SNRs) that emit most of their radiation from a "shell" of shocked material. These shell-type SNRs act through a process called diffusive shock acceleration, whereby particles swept up by the supernova shock waves gain energy by "surfing" the moving shock front and repeatedly crossing it. This mechanism can push charged particles to relativistic velocities, but it's unclear whether the particles can stay in the acceleration region long enough to reach PeV energies. What's more, there is no unequivocal observational evidence that SNRs can produce cosmic rays with energies 
greater than a few tens of $\mathrm{TeV}[3]$.

Other scenarios, however, are possible, and in recent years, gamma-ray observatories have provided a few PeVatron contenders that may help in testing such scenarios. In 2016, the H.E.S.S. Collaboration detected gamma rays of up to tens of TeV coming from the Galactic Center. The researchers interpreted the gamma rays as tracers of $\mathrm{PeV}$ particles and linked them to past activity of Sagittarius $A^{\star}$, the supermassive black hole lurking at the center of our Galaxy [4]. The Tibet AS $\gamma$ Collaboration detected gamma rays up to and above $100 \mathrm{TeV}$ from the direction of the Crab Nebula [5] - a finding that was later confirmed by the High-Altitude Water Cherenkov Observatory (HAWC) and by the Major Atmospheric Gamma Imaging Cherenkov Telescope [6]. Last year, HAWC added several more galactic PeVatron candidates, one of which may be associated with an SNR [7].

Despite this detection bounty, it remains unclear exactly how the parent cosmic rays are accelerated to PeV energies near these gamma-ray sources. The HAWC Collaboration performed a careful analysis of the spatial morphology of the gamma-ray emission from two of their PeVatron candidates, using a multicomponent model to fit their data [8]. They concluded that, in both cases, the observed gamma-ray emission could be explained by ambient gas being hit by cosmic-ray protons that were previously accelerated in star clusters-a hypothesis that challenges the SNR paradigm.

The new result by the Tibet AS $\gamma$ Collaboration adds another piece to the PeVatron puzzle that researchers are trying to complete. The collaboration uses a hybrid detection technique, which combines surface detectors of cosmic-ray-induced air showers and underground muon detectors. The configuration allows them to measure the energy and arrival direction of each gamma ray with an angular resolution of a fraction of a degree for gamma-ray energies of $10 \mathrm{TeV}$ and above. Such features allow the researchers to spot, for the first time, a diffuse "haze" of gamma rays at energies between $100 \mathrm{TeV}$ and $1 \mathrm{PeV}$. The reconstruction of the gamma rays' arrival directions reveal that these diffuse photons arrive from directions that lie within a few degrees of the Galactic Plane (Fig. 2).

By analyzing the gamma-ray spatial distribution and by comparing the observed energy spectrum with theoretical

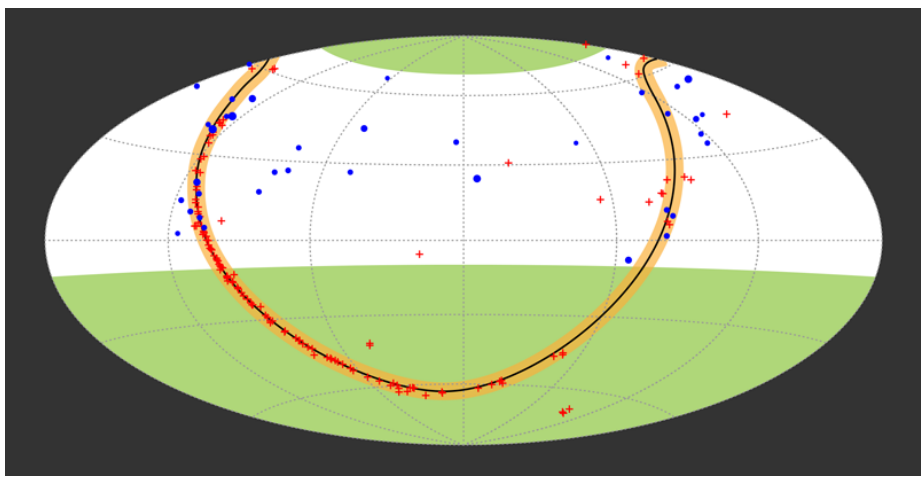

Figure 2: Arrival directions of gamma-ray photons with energies between 0.4 and $1 \mathrm{PeV}$ (blue solid dots). Most detections are clustered in the vicinity of the Galactic Plane (yellow shaded area). The red marks indicate the position of known TeV sources, while the green areas indicate the sky regions outside the field of view of the Tibet AS $\gamma$ observatory.

Credit: M. Amenomori et al. [1]; adapted by APS/Alan Stonebraker

predictions, the researchers test various scenarios for gamma-ray production. First, they conclude that it is unlikely that $\mathrm{PeV}$ electrons, rather than cosmic rays, are producing the gamma rays. By releasing synchrotron radiation as they are accelerated, these electrons would cool too quickly to produce diffuse radiation at energies greater than several hundred TeV. The researchers also show that a scenario involving recently discovered gamma-ray halos around pulsars is unlikely [9]. In this scenario, cosmic-microwave-background photons are converted into gamma-ray photons by scattering off of high-energy electrons and positrons that escaped from a nebula around a pulsar. But the spectrum expected for this case is inconsistent with the data. Finally, the researchers show that a scenario involving PeV cosmic rays produces a good fit to the data, providing a viable explanation. In this scenario, gamma rays predominantly derive from decays of $\pi^{0}$ mesons that are produced as cosmic-ray protons hit interstellar material [10].

With 0.1 - to 1-PeV gamma-ray energies implying 10-times-larger energies for the cosmic-ray progenitors, the observation provides a compelling argument for unidentified galactic PeVatrons. The diffuse nature of the glow is the effect of galactic magnetic fields, which scramble the cosmic rays' original trajectories, impeding the identification of their sources. 
Much more work is needed to put together the PeVatron puzzle, but the advent of new ground-based instruments with much higher sensitivity is ushering in a new era of gamma-ray astrophysics. The latest addition is the Large High Altitude Air Shower Observatory (LHAASO) in China, operational since 2019, which will likely add more PeVatron candidates to the inventory. The Cherenkov Telescope Array, a next-generation, multinational observatory consisting of multiple facilities in Chile and on the Canary Islands, will also hunt for PeVatrons. The Southern Wide-field Gamma-ray Observatory, planned to be constructed in South America, will survey large swaths of the Southern Sky. Because the Solar System is tilted with respect to the Galactic Plane, the Southern Hemisphere has a much better view than the Northern Hemisphere of the Galactic Center-a crowded environment where many more PeVatrons might be discovered.

Petra Huentemeyer: Department of Physics, Michigan

Technological University, Houghton, MI, USA

\section{REFERENCES}

1. M. Amenomori et al. (Tibet AS $\gamma$ Collaboration), "First detection of sub-PeV diffuse gamma rays from the Galactic disk: Evidence for ubiquitous galactic cosmic rays beyond PeV energies," Phys. Rev. Lett. 126, 141101 (2021).

2. F. Aharonian, Very high energy cosmic gamma radiation: A crucial window on the universe (World Scientific, Singapore, 2004).
3. S. Funk, in Handbook of Supernovae, edited by A. W. Alsabti and P. Murdin (Springer, Berlin, 2019), p. 1737.

4. A. Abramowski et al. (H.E.S.S. Collaboration), "Acceleration of petaelectronvolt protons in the Galactic Centre," Nature 531, 476 (2016).

5. M. Amenomori et al. (Tibet AS $\gamma$ Collaboration), "First detection of photons with energy beyond $100 \mathrm{TeV}$ from an astrophysical source," Phys. Rev. Lett. 123, 051101 (2019).

6. V. A. Acciari et al. (MAGIC Collaboration), "MAGIC very large zenith angle observations of the Crab Nebula up to $100 \mathrm{TeV}$," Astron. Astrophys. 635, A158 (2020).

7. A. U. Abeysekara et al. (HAWC Collaboration), "Multiple galactic sources with emission above $56 \mathrm{TeV}$ detected by HAWC," Phys. Rev. Lett. 124, 021102 (2020); A. Albert et al. (HAWC Collaboration), "HAWC J2227+610 and its association with G106.3+2.7, a new potential galactic PeVatron," Astrophys J. Lett. 896, L29 (2020).

8. A. U. Abeysekara et al. (HAWC Collaboration), "HAWC observations of the acceleration of very-high-energy cosmic rays in the Cygnus Cocoon," Nat. Astron. (2021); A. Albert et al. (HAWC Collaboration), "Evidence of $200 \mathrm{TeV}$ Photons from HAWC J1825-134," Astrophys J. Lett. 907, L30 (2021).

9. T. Linden and B. J. Buckman, "Pulsar TeV halos explain the diffuse TeV excess observed by Milagro," Phys. Rev. Lett. 120, 121101 (2018).

10. P. Lipari and S. Vernetto, "Diffuse galactic gamma ray flux at very high energy," Phys. Rev. D 98, 043003 (2018). 